\title{
Expression of Vascular Endothelial Growth Factor on Chondrocytes Increases with Osteoarthritis - An Animal Experimental Investigation
}

\author{
C.O. Tibesku ${ }^{*}, 1$, K. Daniilidis ${ }^{2}$, A. Skwara ${ }^{3}$ and J. Paletta ${ }^{3}$, T. Szuwart ${ }^{4}$ and S. Fuchs-Winkelmann ${ }^{3}$ \\ ${ }^{I}$ Sporthopaedicum Straubing, Germany \\ ${ }^{2}$ Department of General Orthopaedics and Tumororthopaedics, University Hospital Münster, Germany \\ ${ }^{3}$ Department of Orthopaedics and Rheumatology, University Hospital Marburg, Germany \\ ${ }^{4}$ Clinic for Oral and Maxillofacial Surgery, Biomineralisation Working Group, University Hospital Münster, Germany
}

\begin{abstract}
Objective: To evaluate the expression of VEGF by chondrocytes of hyaline cartilage during the course of osteoarthritis (OA).

Methods: In 12 white New Zealand rabbits the anterior cruciate ligament (ACL) was resected to create an anterior instability of the knee. In 12 control rabbits only a sham operation withoutresection of the ACL was done. Four animals of each group were killed at 3,6, and 12 weeks. The load bearing area was evaluated histologically according to Mankin and by immunostaining for VEGF.

Results: In the experimental group, histological grades of OA showed a positive linear correlation with the time after surgery. Immunostaining showed an increased expression of VEGF in the control group after 3 weeks, which dropped to normal after 6 weeks. There was no difference in the progression of OA between control and experimental groups after 3 weeks, but a significant difference was seen after $6(\mathrm{p}=0,01)$ and $12(\mathrm{p}=0,05)$ weeks. A significant positive correlation between VEGF expression and the histological grade of OA was found $(r=0.767 ; \mathrm{p}<0.01)$.

Conclusions: An increase of VEGF expressing chondrocytes occurs during time course of OA.
\end{abstract}

Keywords: VEGF, animal experiments, osteoarthritis.

\section{INTRODUCTION}

Osteoarthritis is a degenerative disease of the hyaline cartilage of the articular surfaces, directly related to ageing and mechanical damage [1], resulting in matrix degradation $[2,3]$. Several studies of late stage arthritis showed that VEGF is expressed in osteoarthritis [4-6], indicating that angiogenesis might be involved in the pathology of OA. In fact, in severe arthritis [7], angiogenesis has been observed. Little is known about the time depended expression of VEGF during the time course of OA. This study was designed to evaluate $(a)$ the time depending expression of VEGF during the course of $\mathrm{OA}$ and $(b)$ possible correlations with histological findings.

\section{MATERIAL AND METHODS}

\section{Animals, Surgery and Macroscopic Evaluation}

The experiments were performed with permission of the local government (G49/2000) in accordance with National Institute of Health (NIH) guidelines as described earlier [8]. Briefly, twenty eight fully grown, female white New Zealand rabbits were used. In the experimental group (12 animals), the anterior cruciate ligament was resected to create instability of the knee. In the control group (12 animals),

\footnotetext{
*Address correspondence to this author at the Sporthopaedicum Straubing, Bahnhofplatz 8, D-94315 Straubing, Germany; Tel: +49 - 9421 - 9957 0; Fax:+49 - 9421 - 9957 399; E-mail: carsten@tibesku.de
}

only a sham operation without resection of the ACL was done. Four animals of each group were killed at 3, 6, and 12 weeks postoperatively. In addition, four animals ( 0 weeks) did not undergo any treatment and were killed at the time of surgery. After slaying the animals, both distal femurs were prepared and immediately stored at $-80^{\circ} \mathrm{C}$. Macroscopic grading of arthritis was done as described previously [8].

\section{Histological Evaluation}

Sample preparation and immunostaining were performed as described earlier [8], with some modifications. Briefly, frozen tissue samples were thawed, fixed with $4 \%$ paraformaldehyde, decalcificated using buffered EDTA $(20 \%, \mathrm{pH} 7.4)$, dehydrated and embedded in paraffin. Sections $(5 \mu \mathrm{m}$ thick) were cut, mounted on poly-L-lysine coated glass slides, deparaffinized in xylene, and washed. Epitope unmasking was done using proteinase $\mathrm{K}$ digestion (DAKO, Germany) for 5 minutes and endogenous peroxidase activity was quenched by treating tissue sections with $3 \% \mathrm{H}_{2} \mathrm{O}_{2}$ for 10 minutes, non-specific binding was blocked using 3\% bovine serum albumin ( $\mathrm{BSA} /$ TrisHCl), each followed by an excessive washing procedure. Sections thus prepared were incubated with monoclonal mouse IgG antibodies against VEGF diluted 1:50 in Tris/BSA (Calbiochem Clone JH 121) at $4^{\circ} \mathrm{C}$ over night. Visualization was done using EnVision+ System- HRP with AECchromogene (DAKO Envision Systems, Germany, K4001). As control instead of primary antibody, mouse IgG1 with irrelevant specificity (Aspergillus niger glucose oxidase, 
DAKO, Germany) was used. In addition, sections were incubated without primary antibody.

\section{Image Analysis}

Histological sections were photographed (CoolSnap-Pro Color A00J82025, Media Cybernetics, Silver Spring, MD, USA). The cells were counted using the Image-Pro Plus software for Windows, version 4.1 (Media Cybernetics, Silver Spring, MD, USA). The total number of chondrocytes was determined in a hematoxylin and eosin stained section and compared with the number of immunohistochemically stained cells.

Histological grade of OA was evaluated according to Mankin [9] by two independent investigators who were unaware of the source of the specimens. The sections were decalcified by using EDTA which leads to an adapted mankin score which we used in the present study.

\section{Statistical Analysis}

Statistical analysis was performed using SPSS 11.0 (SPSS GmbH, Munich, Germany). Student's $t$ test was used for comparison of scores, and Spearman's coefficient was calculated for correlations. $\mathrm{P}$ values less then 0.05 were considered significant.

\section{RESULTS}

Two animals developed postoperative hematomas, first a superficial wound infection treated with a single shot antibiotic, and second animal wound gap re-operated on 2 days after surgery. All four animals were included in the analysis. At the time of death, one animal of group one had intact ACLs and was excluded from evaluation. In total, 54 joints were evaluated.

\section{Histological Grading of OA}

According to the Mankin score, the experimental group showed a highly significant increase in the grade of OA in comparison with the control group and the untreated group (Figs. 3-6). In the untreated group the mean (SD) grade of OA was 1.75 (1.58). In the control group it was $3.25(0.71)$ after 3 weeks, 1 (1.20) after 6 weeks, and 2.25 (1.17) after 12 weeks. In the experimental group, the scores were 7.25
(3.62) after 3 weeks, 8 (2.88) after 6 weeks, and 9.83 (3.06) after 12 weeks. The experimental group had significantly higher values than the control group at every point of time $(p<0.01)$. The average histological grade of OA increased with the number of postoperative weeks. It is remarkable that in the control group the histological grading increases within the first three weeks and drops to normal in the following time.

\section{Immunohistochemical Staining of VEGF}

Chondrocytes expressing VEGF were found in the experimental and control groups (Fig. 1). In the control group, the mean percentage (SD) of VEGF positive cells was $16.2 \pm 4.7 \%$ after 3 weeks, $11.5 \pm 5.9 \%$ after 6 weeks, and $11.6 \pm 3.3 \%$ after 12 weeks. In the experimental group, percentages were $17.7 \pm 5.7 \%$ after 3 weeks, $19.4 \pm 4.6 \%$ after 6 weeks, and $21.3 \pm 5.4 \%$ after 12 weeks (Fig. 1). There was no significant difference between control and experimental groups after 3 weeks $(p=0.594)$, but a significant difference was seen after 6 and 12 weeks $(p=0.01$ and $\mathrm{p}=0.05$ ). It is remarkable that in the control group the percentage of VEGF expressing chondrocytes increases within the fist three weeks and drops to normal in the following time.

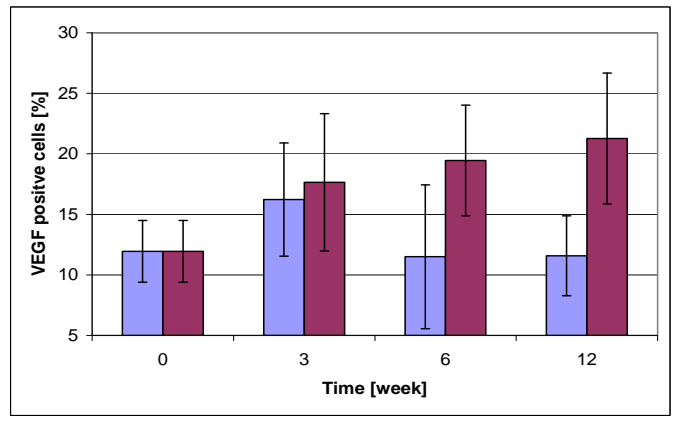

Fig. (1). Time course of the development of VEGF expression in osteoarthritis (light bar presents the sham operation, grey bar the acl resection group). The percentage of VEGF expressing chondrocytes was significantly increased in the ACL resection group after 6 $(p=0.01)$ and $12(p=0.05)$ weeks. Data represent mean \pm SD from 4 independent determinations.
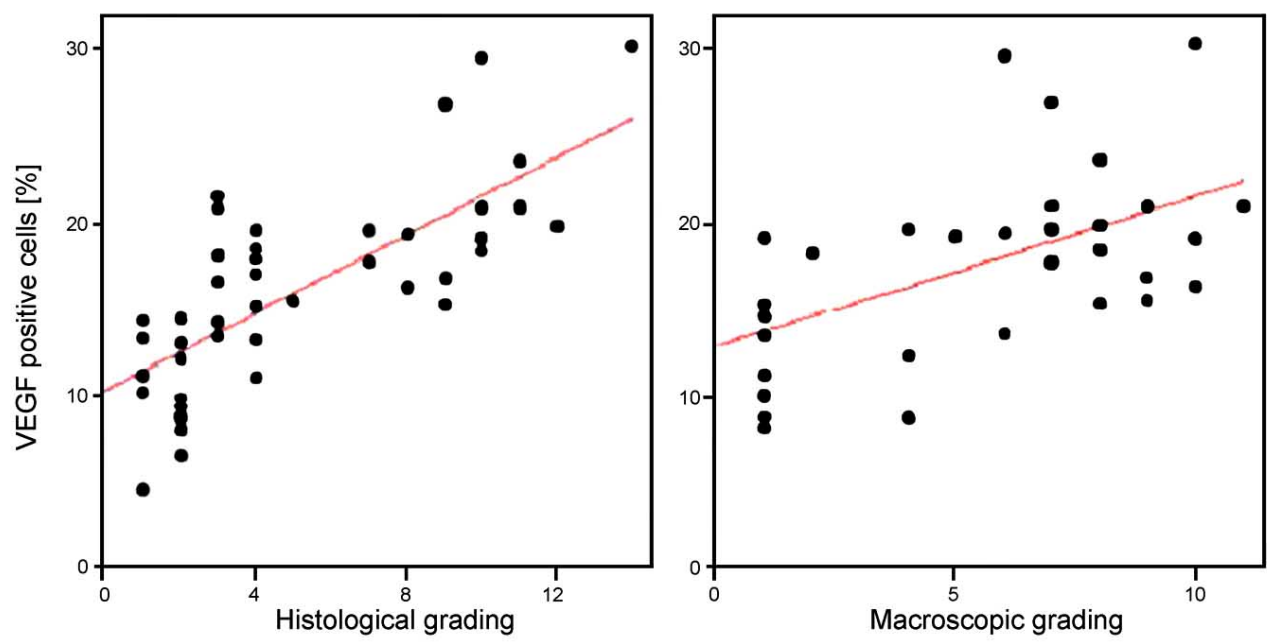

Fig. (2). Correlation between the histological and macroscopic grade of osteoarthritis (OA) with expression of VEGF by chondrocytes. Significant positive correlations were found with a Spearman's coefficient of $0.767(\mathrm{p}=0.01)$ and $0.518(\mathrm{p}=0.02)$. 


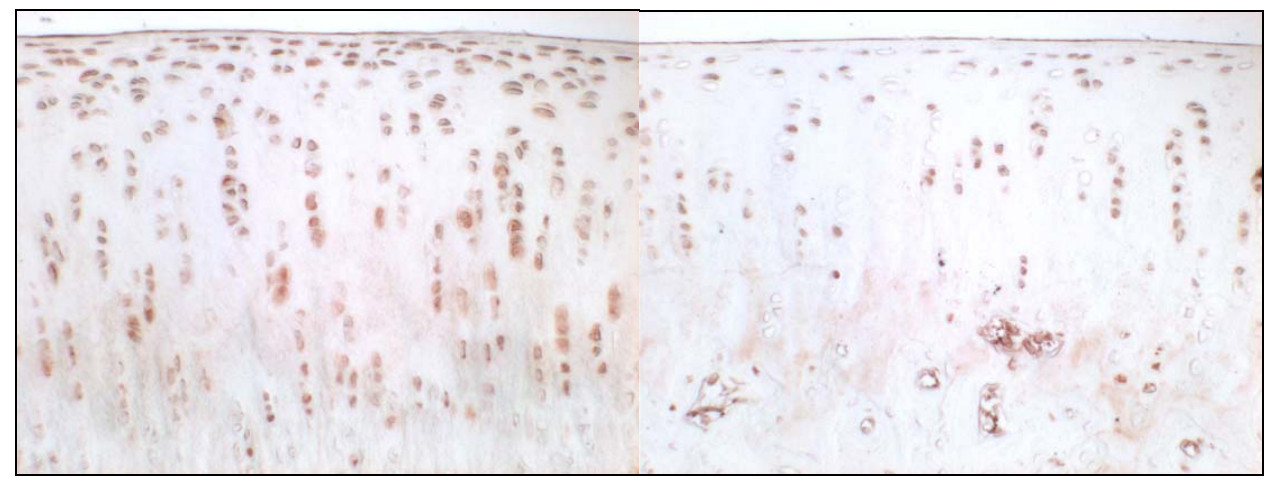

Fig. (3). Immunohistochemical staining of VEGF, 3 weeks after sham operation (left side) and 3 weeks after resection of the anterior cruciate ligament (right side).

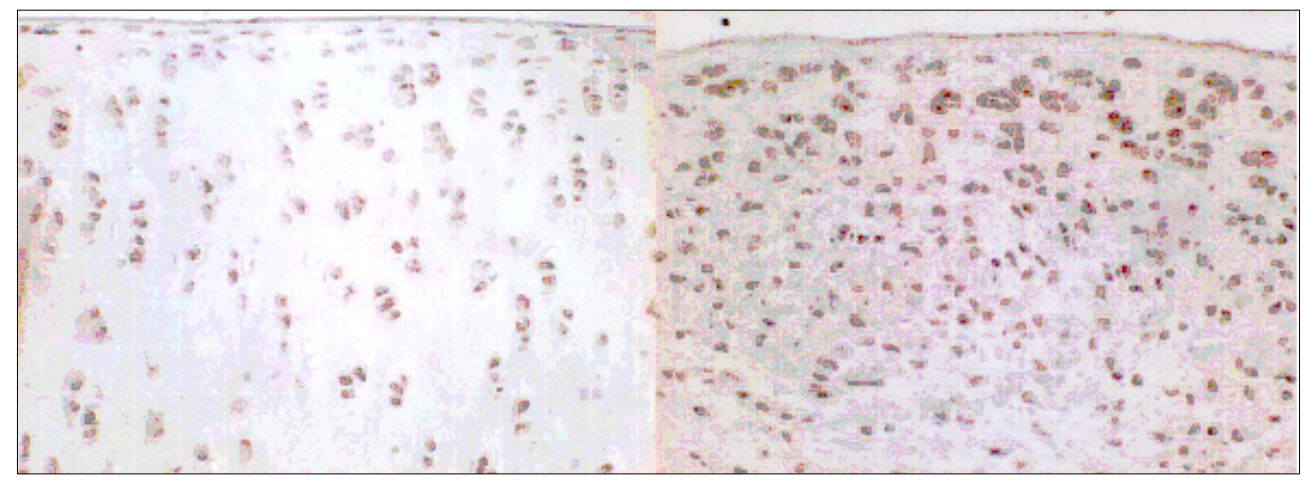

Fig. (4). Immunohistochemical staining of VEGF, 6 weeks after sham operation (left side) and 6 weeks after resection of the anterior cruciate ligament (right side).

A significant correlation was found between the percentage of VEGF positive chondrocytes and the histological grade $(r=0.767, \mathrm{p}=0.01)$ as well as the macroscopic grade $(r=0.518, \mathrm{p}=0.02)$ (Fig. 2). Focusing on osteophyte formation (as part of the macroscopic grade) a positive correlation $(r=0.651 ; p<0.01)$ was detectable. The VEGF staining occurred throughout the cartilage.

\section{DISCUSSION}

The present study demonstrates that VEGF is synthesized by chondrocytes of articular cartilage in knees of healthy rabbits. With the development of osteoarthritis due to ACL resection this ratio alters, resulting in an increase of VEGF expressing chondrocytes, correlating with the degree of osteoarthritis. Therefore, the expression of VEGF might be a useful marker of OA. Our data, obtained in an experimental animal model, corresponds with findings of others [4-6, 10, 11] who found that VEGF expression is up regulated in human chondrocytes during late stage $\mathrm{OA}$.

Osteophyte formation and extension of the subchondral growth plate have been observed in OA and involve processes very similar to enchondral ossification, which is highly dependent on VEGF [12]. In our studies, the amount of VEGF producing chondrocytes was positively correlated with the osteophyte formation. A methodological limitation of our study is that we mixed the values of sham and ACLT in the statistic which could influence the present results.

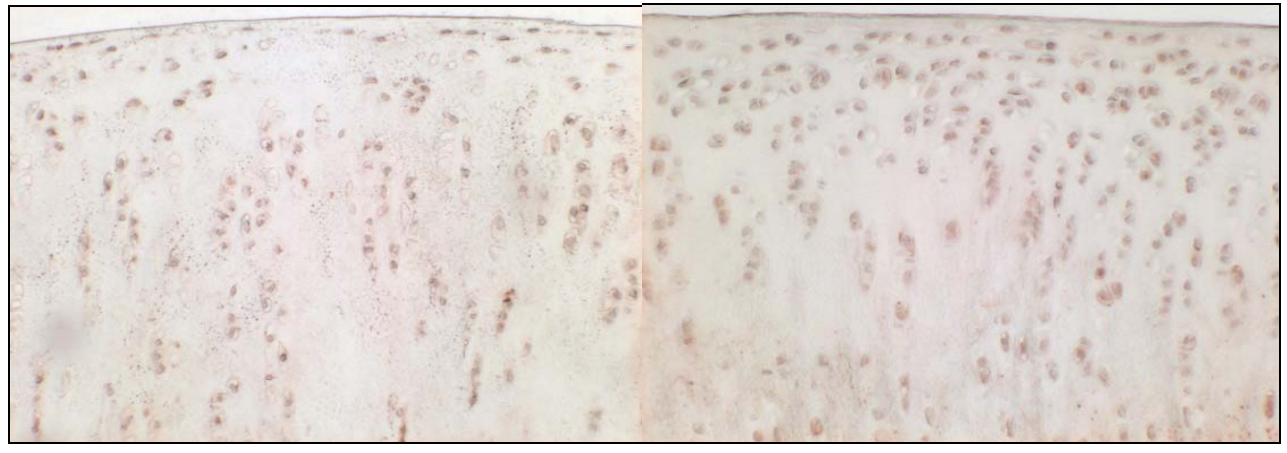

Fig. (5). Immunohistochemical staining of VEGF, 12 weeks after sham operation (left side) and 12 weeks after resection of the anterior cruciate ligament (right side). 


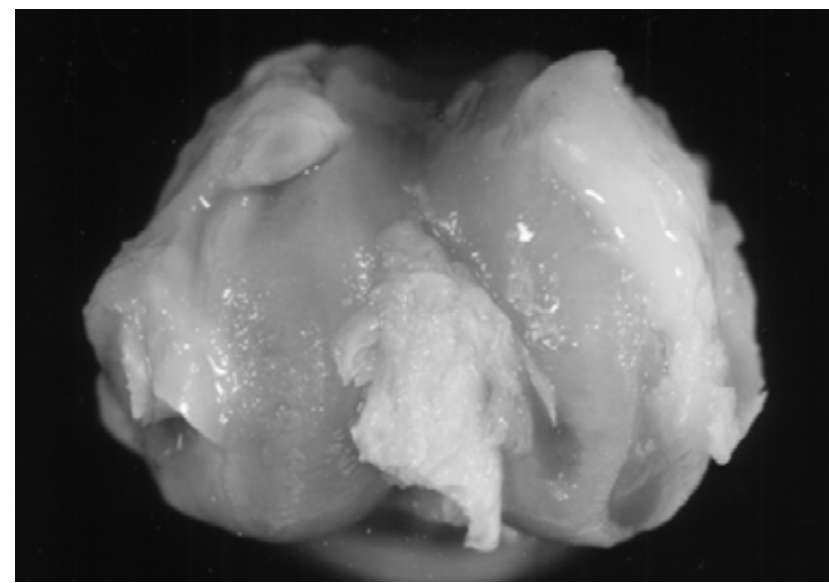

Fig. (6). 12 weeks after acl resection showing pronounced osteophytes and exposure of the subchondral cartilage zone.

Focusing on the time course of the control group, three weeks after surgery the percentage of VEGF expressing chondrocytes increases although no mechanic instability was induced by ACL resection. This might be due to angiogenic process common in wound healing $[13,14]$. Nevertheless, it is remarkable that the histological grading according to Mankin is raised after 3 weeks and drops to normal in the following time, comparable to the percentage of VEGF expressing chondrocytes. This might be interpreted that VEGF alone has a negative impact on the integrity of the articular cartilage as postulated by Pufe [15]. This finding has far reaching consequences; for antiangiogenic therapy might be expedient in the treatment of osteoarthritis. Further studies are needed to evaluate VEGF as a target in osteoarthritis treatment.

\section{ACKNOWLEDGEMENT}

We thank Ms S, Kupich for expert technical assistance.

\section{ABBREVIATIONS}

$\begin{array}{ll}\mathrm{ACL} & =\text { Anterior cruciate ligament } \\ \mathrm{OA} & =\text { Osteoarthritis } \\ \mathrm{VEGF} & =\text { Vascular endothelial growth factor }\end{array}$

\section{REFERENCES}

[1] Hough A. Pathology of osteoarthritis. $12^{\text {th }}$ ed. Philadelphia: Lea \& Febinger 1993.

[2] Malemud CJ. Fundamental pathways in osteoarthritis: an overview. Front Biosci 1999; 4: D659-61.

[3] Lohmander LS. Articular cartilage and osteoarthrosis. The role of molecular markers to monitor breakdown, repair and disease. J Anat 1994; 184(Pt 3): 477-92.

[4] Pufe T, Petersen W, Tillmann B, Mentlein R. The splice variants VEGF121 and VEGF189 of the angiogenic peptide vascular endothelial growth factor are expressed in osteoarthritic cartilage. Arthr Rheumat 2001; 44(5): 1082-8.

[5] Pfander D, Kortje D, Zimmermann R, et al. Vascular endothelial growth factor in articular cartilage of healthy and osteoarthritic human knee joints. Ann Rheum Dis 2001; 60(11): 1070-3.

[6] Enomoto H, Inoki I, Komiya K, et al. Vascular endothelial growth factor isoforms and their receptors are expressed in human osteoarthritic cartilage. Am J Pathol 2003; 162(1): 171-81.

[7] Bullough PG, Jagannath A. The morphology of the calcification front in articular cartilage. Its significance in joint function. J Bone Joint Surg Am 1983; 65(1): 72-8.

[8] Tibesku CO, Szuwart T, Ocken SA, Skwara A, Fuchs S. Expression of the matrix receptor CD44v5 on chondrocytes changes with osteoarthritis: an experimental investigation in the rabbit. Ann Rheum Dis 2006; 65(1): 105-8.

[9] Mankin HJ, Dorfman H, Lippiello L, Zarins A. Biochemical and metabolic abnormalities in articular cartilage from osteo-arthritic human hips. II. Correlation of morphology with biochemical and metabolic data. J Bone Joint Surg Am 1971; 53(3): 523-37.

[10] Hayami T, Funaki H, Yaoeda K, et al. Expression of the cartilage derived anti-angiogenic factor chondromodulin-I decreases in the early stage of experimental osteoarthritis. J Rheumatol 2003; 30(10): 2207-17.

[11] Zhou JL, Liu SQ, Qiu B, Hu QJ, Ming JH, Peng H. Effects of hyaluronan on vascular endothelial growth factor and receptor-2 expression in a rabbit osteoarthritis model. J Orthop Sci 2009; 14(3): 313-9.

[12] Gerber HP, Vu TH, Ryan AM, Kowalski J, Werb Z, Ferrara N. VEGF couples hypertrophic cartilage remodeling, ossification and angiogenesis during endochondral bone formation [see comment]. Nat Med 1999; 5(6): 623-8.

[13] Grazul-Bilska AT, Johnson ML, Bilski JJ, et al. Wound healing: the role of growth factors. Drug Today 2003; 39(10): 787-800.

[14] Frank S, Hubner G, Breier G, Longaker MT, Greenhalgh DG, Werner S. Regulation of vascular endothelial growth factor expression in cultured keratinocytes. Implications for normal and impaired wound healing. J Biol Chem 1995; 270(21): 12607-13.

[15] Pufe T, Kurz B, Petersen W, et al. The influence of biomechanical parameters on the expression of VEGF and endostatin in the bone and joint system. Anna Anat 2005; 187(5-6): 461-72. 